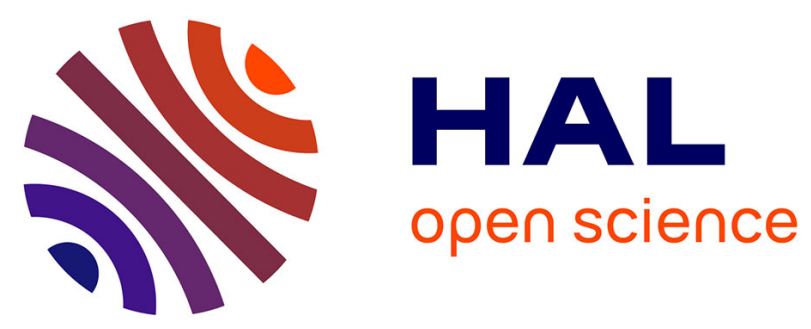

\title{
Provenance et circulation de la céramique médiévale
} Jacques Thiriot

\section{To cite this version:}

Jacques Thiriot. Provenance et circulation de la céramique médiévale. Bulletin de la Société préhistorique française, 1991, Du Terrain au laboratoire. Pour un meilleur dialogue en archéologie. Colloque SPF-GMPCA, Paris, 1989, 86 (10-12), pp.343-346. halshs-01400128

\section{HAL Id: halshs-01400128 \\ https://shs.hal.science/halshs-01400128}

Submitted on 21 Nov 2016

HAL is a multi-disciplinary open access archive for the deposit and dissemination of scientific research documents, whether they are published or not. The documents may come from teaching and research institutions in France or abroad, or from public or private research centers.
L'archive ouverte pluridisciplinaire HAL, est destinée au dépôt et à la diffusion de documents scientifiques de niveau recherche, publiés ou non, émanant des établissements d'enseignement et de recherche français ou étrangers, des laboratoires publics ou privés.

\section{(1) $\$(0)$}

Distributed under a Creative Commons Attribution - NonCommercial - ShareAlikel 4.0 


\section{Bulletin}

\section{de la SOCIÉTÉ \\ PREHISTORIQUE FRANÇAISE \\ 1989 - TOME 86 numéro 10 - 12}

\section{Actes de la séance SPF-GMPCA du CPF Paris, 1989}

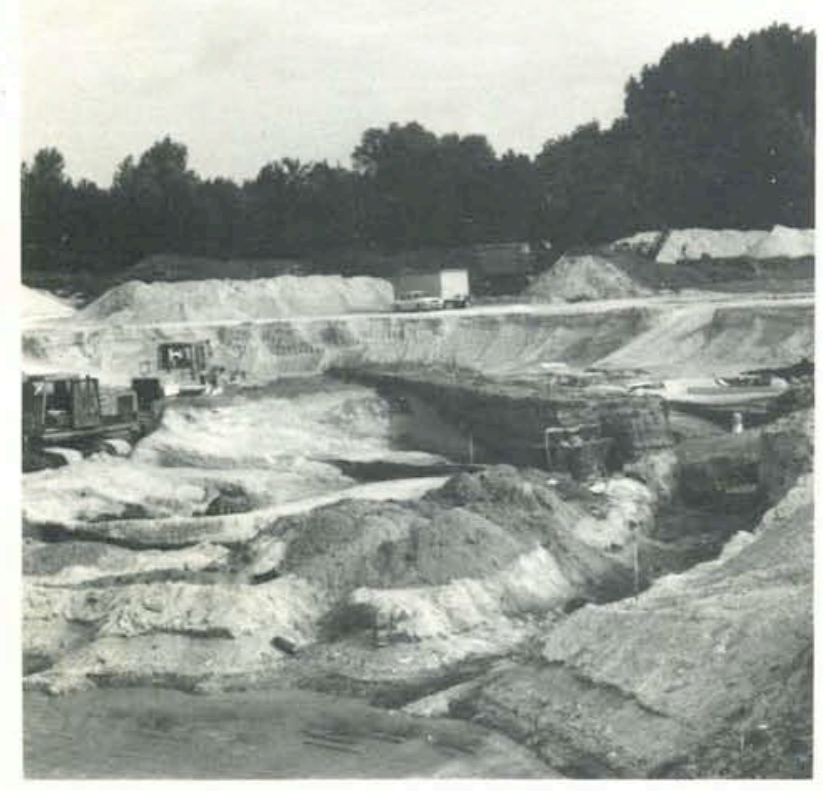

\section{DU TERRAIN...}
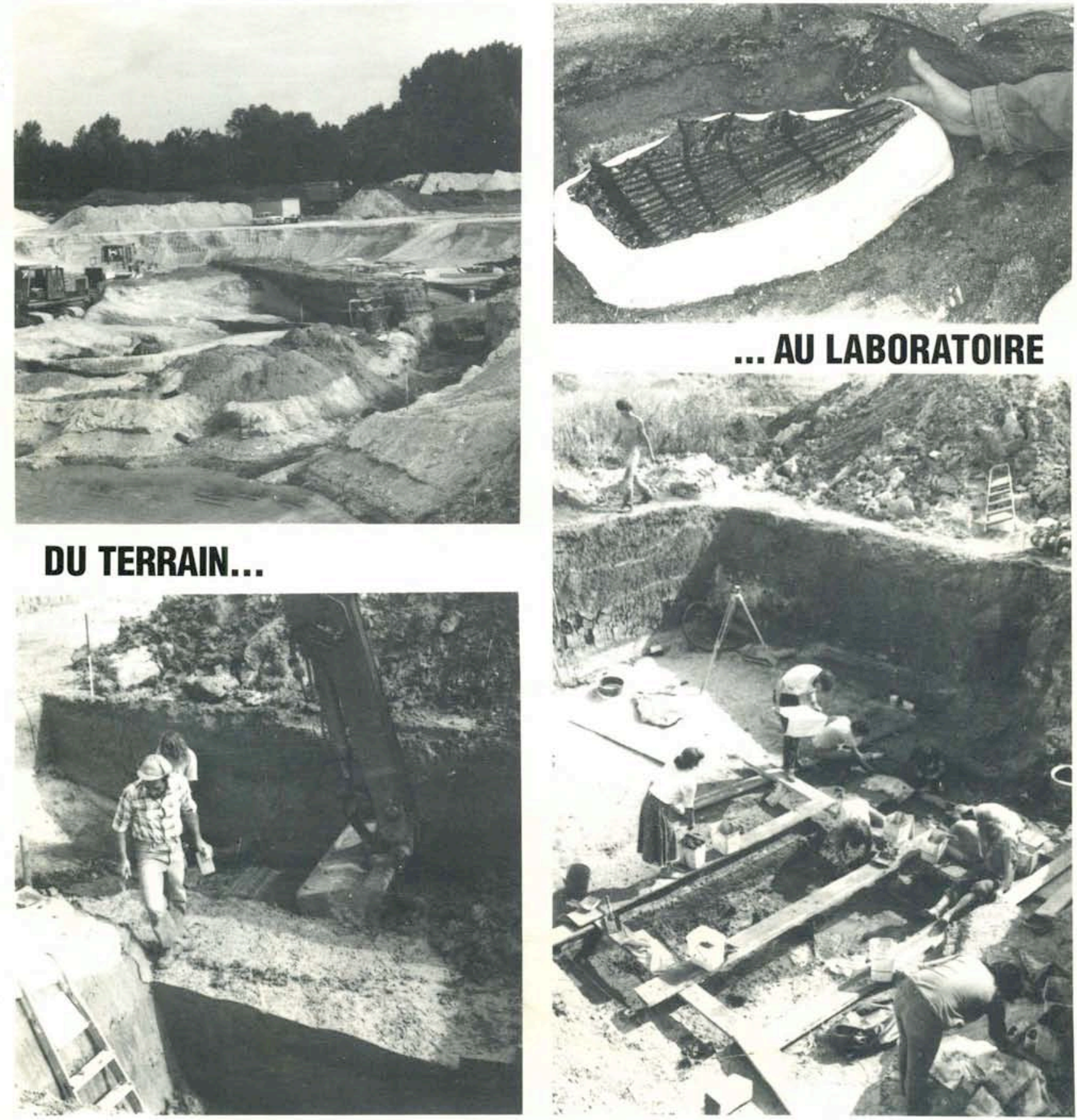

\section{AU LABORATOIRE}

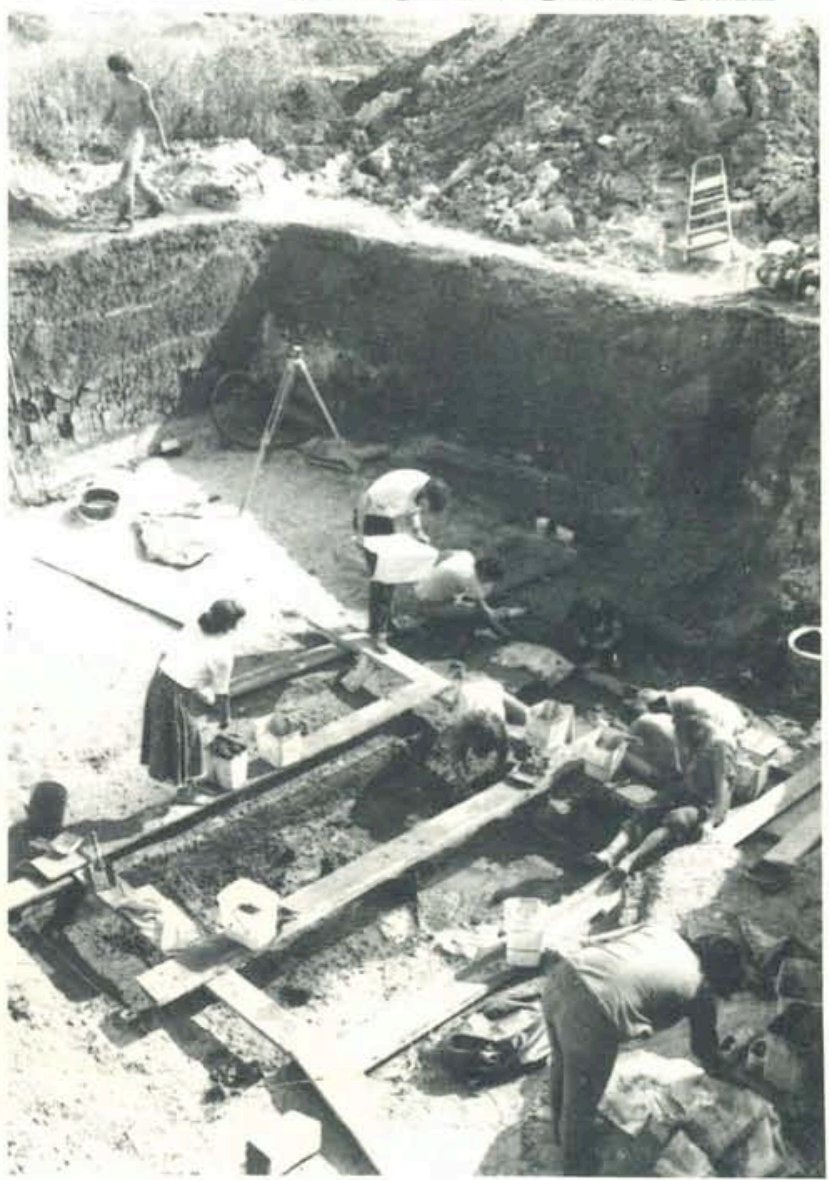

Publié avec le concours du Centre $N$ ational de la Recherche $S_{\text {cientifique }}$

et du Ministère de la Culture et de la Communication (Sous-Direction de l'Archéologie et Direction des Musées de France) 


\title{
Bulletin \\ de la SOĆIÉTÉ \\ PRÉHISTORIQUE \\ FRANÇAISE
}

Sommaire du numéro 10 - 12 : Actes de la séance SPF-GMPCA du Congrès Préhistorique de France - Paris, 1989

\section{DU TERRAIN AU LABORATOIRE : POUR UN MEILLEUR DIALOGUE EN ARCHÉOLOGIE}

\author{
Sous la direction de
}

Jean-Denis Vigne, Michel Menu, Catherine Perlès et Hélène Valladas

J.-P. Mohen et J. Évin

Préface : L'archéométrie, tradition de la méthode scientifique préhistorique ou innovation de la nouvelle archéologie?

J.-D. Vigne, M. Menu, C. Perlès et H. Valladas Introduction

C. Perlès

Points de vue sur l'archéométrie
M. Martinaud et G. Colmont

De la coopération entre partenaires pour les re-

\section{PREMIÈRE PARTIE : PERSPECTIVES ANALYTIQUES}

QUELLE STRATÉGIE POUR UNE REPRÉSENTATIVITÉ OPTIMALE DES STRUCTURES ANCIENNES?

A. Ferdière

Fouilles de "sauvetage ", fouilles de " recherche ", l'impossible opposition

$$
\text { Publie avec leconcours du C }
$$

Publié avec le concours du $C$ cherches géophysiques de structures archéologiques

\section{Perlès}

Discussions

INTÉGRER LES DIFFÉRENTES IMAGES DE L'ENVIRONNEMENT DANS L'ESPACE ET LE TEMPS?

\section{J. Guilaine}

Interrogation sur les essais de modélisation de l'environnement à partir de stratigraphies néolithiques sous abris : l'exemple de Font-Juvénal

D. Mordant

Intégrer les différentes images de l'environnement dans l'espace et le temps en milieu fluviatile : exemple de la Petite-Seine

J.-D. Vigne

Discussions

entre $N$ ational de la Recherche $S$ cientifique et du Ministère de la $C_{\text {ulture et de la }} C_{\text {ommunication }}\left(S_{\text {ous- }} \mathrm{D}_{\text {irection de l'A }}\right.$ Archéologie et $\mathrm{Direction}$ des Musées de France) 


\section{COHÉRENCE DES DIFFÉRENTES MÉTHODES DE DATATION}

L. Meignen et al.

Datations relatives, datations absolues : les populations du Paléolithique moyen au Proche-Orient

A. Bocquet

Cohérence entre les dates dendrochronologiques alpines au Bronze final et la chronologie typologique italique

H. Valladas

Discussions

DE L'ARCHÉOMÉTRIE AUX SYSTĖMES TECHNIQUES D'UNE SOCIÉTÉ

J. Thiriot

Provenance et circulation de la céramique médiévale

M. Menu, Ph. Walter

Analyse des matériaux et systèmes de production

M. Menu

Discussion

\section{DEUXIÈME PARTIE : INTÉGRATION DES APPROCHES, EXPÉRIENCES ET RÉSULTATS}

\section{PALÉOLITHIQUE ET MÉSOLITHIQUE}

L. Meignen et al.

Néandertaliens et hommes modernes au ProcheOrient : chronologie et comportements culturels

M.-R. Séronie-Vivien

Un habitat en grotte : la grotte de Pégourié (Caniacdu-Causse, Lot)

M.-C. Marinval-Vigne, J.-D. Vigne et coll.

Noyens-sur-Seine, site stratifié en milieu fluviatile: une étude multidisciplinaire intégrée

N. Petit-Maire

L'anthropoclimatologie: du passé à l'avenir de l'Homme
R.J. March et al.

Étude des structures de combustion archéologiques d'Argentine

\section{NÉOLITHIQUE ET AGE DU BRONZE}

J.-F. Bouchard

Archéologie préhispanique du littoral pacifique nordéquatorial

B. Briard et al.

Archéologie et environnement en forêt de Brocéliande, un exemple d'étude pluridisciplinaire

J.-P. Mohen et coll.

Ateliers métallurgiques dans l'habitat protohistorique du Fort-Harrouard

F. Debaine, H.P. Francfort

Réseau d'irrigation et cultures protohistoriques en Asie

M. Wuttmann

Bilan des contributions physiques et chimiques à l'étude du site de Balat (Egypte)

\section{AGE DU FER ET PÉRIODES HISTORIQUES}

M. Lamotte, A. Marliac

Des structures complexes résultant de processus naturels et anthropiques : exemple du tertre de Mongossi au Nord-Cameroun

J.-P. Bravard, J. Burnouf, A. Vérot

Géomorphologie et archéologie dans le bassin du Rhône : questions et réponses d'un dialogue

F. Trément

La région des Étangs de Saint-Blaise: pour une approche archéologique et paléoécologique d'un milieu de vie

M. L'Hour

Un exemple du développement de l'approche pluridisciplinaire en archéologie sous-marine: l'épave Aber Wrac'h 1

370 J. Thiriot

Ateliers de potiers en Uzège et Bas-Rhône : apport des recherches archéologiques et de laboratoire 


\title{
Provenance et circulation de la céramique médiévale
}

\author{
par Jacques Thiriot
}

M'entendre parler d'un tel sujet paraîtra quelque peu curieux et pour le moins étonnant à ceux qui connaissent mes recherches il est vrai dédiées à la production potière mais toutefois un peu éloignées du problème spécifique de la provenance. Cette démarche ne m'est bien évidemment pas étrangère puisqu'en quelque sorte j'apporte des références (carrières d'argiles et productions datées) à l'analyste. Bien d'autres ussent été plus compétents, je pense aux « hommes de l'art » (Picon, 1989) ou aux archéologues qui collaborent étroitement et depuis longtemps à ce type de recherche (1).

Mon propos en étroite liaison avec ma recherche des ateliers de potiers médiévaux et modernes, de plus en plus collective et pluridisciplinaire, est donc surtout axé sur la période médiévale qui ici, en quelque sorte, cumule les problématiques des périodes antérieures en ajoutant les données textuelles et une application peut-être plus évidente des données ethnoarchéologiques. Le renouveau spectaculaire, ces dix dernières années dans de nombreuses régions, des études sur la céramique médiévale, et ses moyens de production, justifie ce choix pour qu'enfin une politique de recherche archéométrique soit appliquée aux périodes médiévales. Mon adhésion au GMPCA depuis sa création et surtout mon appartenance à un laboratoire d'archéologie qui " utilise » couramment l'archéométrie depuis longtemps m'entraîne à considérer la collaboration archéologue/archéomètre comme naturelle et évidente.

(1) M. Picon a accepté de guider mes pas en ce domaine.
UN EXEMPLE : L'ÉTUDE ARCHÉOLOGIQUE ET DE LABORATOIRE DE LA CERAMIQUE DE ROUGIERS $X I I^{e}-X V^{e} S$.

On se reportera très utilement à cet exemple (éliminé ici par manque de place) de collaboration archéologue/archéomètre pour illustrer mes propos généraux (Démians d'Archimbaud, 1987).

LA CÉRAMIQUE DANS LA RECHERCHE DU MÉDIÉVISTE

Si la céramique peut être considérée comme un de ses matériaux, le médiéviste n'en trouve guère mention sauf quelques rares exceptions dans des inventaires souvent tardifs. L'historien du monde artisanal a plus de chance surtout à partir du $\mathrm{XIV}^{\mathrm{e}} \mathrm{s}$. quant à l'organisation du métier, la société artisanale et son cadre de vie, la commercialisation et l'importation, les transferts de technologie par déplacement des potiers... L'archéologue en a fait son principal fossile directeur : indice majeur de datation, un des critères matériels du mode de vie et de l'occupation de l'espace habité mais aussi révélateur de société et d'échanges économiques. Fouillant les ateliers, l'archéologue retrace tous les aspects matériels de sa fabrication tout en fixant des repères chronologiques sûrs en théorie. L'analyse en stratigraphie des différentes masses de céramiques en présence et surtout l'étude typologique périodisée classique en comparaison avec les découvertes d'autres sites bien strati- 
fiés permet une approche sérieuse de la vaisselle employée et de son évolution dans le temps mais aussi, par comparaison toujours quelque peu subjective, la formulation d'hypothèses concernant l'origine et par conséquence la circulation de ces produits. Cette typologie introduit une vision globale sur la longue durée en même temps qu'elle met l'accent sur les disparités régionales et chronologiques. A ce stade, l'étude sera d'autant plus fiable que le fond étudié sera bien stratifié et bien jalonné par des éléments datables mais surtout que le céramologue sera bien formé aux méthodes de travail sur un tel matériau (en particulier traitement de grandes masses), sera conscient des limites des «manipulations statistiques " et aura une connaissance très élargie des recherches parallèles dans une aire géographique forcément très large recouvrant les domaines commerciaux en présence. Pour le Midi méditerranéen, cela englobe l'Italie à l'Espagne, le Maghreb et même le Proche-Orient... Pour l'historien et l'archéologue associés dans la recherche de provenance, la simple étude de matériel peut être largement prolongée par une recherche régressive à partir de l'étude des ateliers récents (textes, tradition orale, étude des cartes et terrain). Cette démarche relativement nouvelle (environ une dizaine d'années) est maintenant assez bien développée.

LA DEMANDE DES ARCHÉOLOGUES (CÉRAMOLOGIE DE LABORATOIRE)

Leur curiosité n'est pas nouvelle (température de cuisson, dureté, porosité) et s'accompagne parfois de "perversions" (le besoin de scientisme pour la publication qui relègue en annexe une " expertise " avec des tableaux de chiffres, une courbe ou un histogramme) signe de manque de maturité. La recherche se structurant et devenant le fait d'équipes de plus en plus élargies (inter-périodes), les problématiques s'affirment, les demandes n'en sont que plus précises et impératives. Elles peuvent avoir plusieurs aspects complémentaires hormis le traitement statistique des données courantes:

- technologie de fabrication de l'objet et sa situation dans l'évolution plus ou moins bien connue des techniques: vers une Histoire des Techniques...;

- vérification des classifications archéologiques et mise en évidence de cas particuliers ;

- enfin recherche de provenance débouchant naturellement sur les problèmes de circulation des produits : circuits commerciaux, échanges.
Dans la démarche archéologique la plus rigoureuse possible subsiste toujours une part plus ou moins grande de subjectivité que l'archéologue espère lever en faisant appel à l'archéomètre, l'homme de science qui est sensé employer des méthodes plus exactes.

MÉTHODES EN CÉRAMOLOGIE DE LABORATOIRE

Les méthodes ne manquent pas: pétrographie, minéralogie, fluorescence des rayons $\mathrm{X}$, activation neutronique, spectrographie optique d'émission, absorption atomique. Le choix de la méthode est souvent affaire d'opportunité ; chacune ayant sa spécificité, ses avantages et inconvénients. Le débat est ici une affaire de spécialiste... Les méthodes géochimiques sont sans doute les plus employées et, parmi elles, la fluorescence $\mathrm{X}$.

Mon propos n'est pas de développer ici les procédures, leurs bienfaits et leurs embuches. Peut-être faut-il rappeler seulement l'importance de l'établissement des preuves qui, elles seules - par la constitution d'un échantillonnage plus conséquent, la mesure des ressemblances et leur interprétation - rendent crédible la détermination d'origine.

LA DEMANDE DES ARCHÉOMÈTRES (CÉRAMOLOGIE DE LABORATOIRE)

\section{- Pour répondre à la demande des archéologues :}

- nécessité d'une archéologie structurée au niveau de la région ayant suffisamment de recul par rapport aux urgences et dotée de véritables programmes de recherche ;

- besoin de typologies périodisées bien établies si possible sur la longue durée ;

- besoin de recherches sur la longue durée à propos des centres de production récents par étude de terrain et des textes ;

- besoin surtout de questions bien formulées ayant une incidence historique et s'affranchissant d'une certaine inertie répétitive.

\section{- Pour leur propre demande :}

— besoins d'exemples ou de problèmes archéolo- 
giques pour mettre au point et roder de nouvelles procédures ou méthodes de recherche ;

- pour certains, besoin d'applications spectaculaires ou de prestige (déviation ?) : archéomètres comme archéologues sont parfois victimes d'une politique du spectaculaire, du médiatique... ;

- besoin enfin d'une collaboration la plus large possible pour une recherche réellement pluridisciplinaire. Cette collaboration s'accompagne naturellement d'un besoin de dialogue entre disciplines complémentaires où chacun argumente dans son propre domaine et questionne l'autre ;

- l'archéométrie "peut se décrire, à la fois, comme la face cachée et le point de passage obligé de la discipline archéologique. L'archéomètre est clairement invité à ne pas se considérer comme un archéologue supérieur, mais la référence [...] à son travail est devenue comme une obligation statutaire... " (Ligot, 1989, 465). Les deux ont besoin d'une formation à la discipline de l'autre afin d'amoindrir la barrière du langage et posséder des notions précises des démarches, méthodes de travail et de traitement des données, d'où l'importance de la formation permanente...

UNE SITUATION DE CRISE EN CÉRAMOLOGIE

\section{- Déséquilibres en archéologie :}

- Le poids grandissant des urgences imposées par les grands travaux et des grandes masses de matériel à traiter limite la recherche programmée et sollicite de plus en plus le chercheur qui a du mal à répondre faute de structures officielles, de moyens (en crédits et en temps), et de personnels formés. Situation de crise aux dépens de la recherche programmée toutefois indispensable et qu'il importe de sauvegarder en priorité.

- Le poids, la crédibilité variables accordés aux archéologues des différentes périodes de l'Histoire, aux différents programmes ou objets de recherche, enfin à l'appartenance des archéologues aux différentes instances nationales ou territoriales (ou même aux " amateurs ») nuit à une véritable politique de recherche archéologique.

- Pour certains archéomètres, le problème viendrait du manque de problématique, du manque de maturité des archéologues face à leur discipline. A voir... On pourrait ajouter que la dispersion et le cloisonnement des archéologues n'arrange rien. Ces travers, s'ils existent encore par endroits, sont certainement en forte régression; des regroupements transversaux font disparaître totalement les cloisonnements de tout ordre.

- Il manque toujours une politique cohérente (une structure ?) de l'archéologie de recherche digne de ce nom ayant suffisamment pris conscience de ses besoins spécifiques (en dehors des problèmes que pose l'archéologie de sauvetage) et surtout dotée décemment de crédits spécifiques des différentes institutions.

\section{- Dispersion et moyens des laboratoires}

Des laboratoires éparpillés, de recherche et non de service nés et maintenus au hasard d'initiatives personnelles, sont dotés d'un matériel performant mais sont dénués de crédits correspondants. Le manque de structuration à défaut de regroupement limite l'efficacité des équipes (Est-ce un manque de personnel ? La céramologie est la mieux dotée à ce sujet...). Le manque de structure de continuité est sans doute plus grave aboutissant à terme à la fermeture de bon nombre d'entre eux. Les maigres crédits sont alors dirigés vers la recherche sur les méthodes au détriment des applications ("service » ou mieux collaboration à des programmes d'archéologues qui nécessite malheureusement plus de crédits). De rares contrats, obtenus à quel prix !, ne remplacent pas, à défaut de véritable Programme interdisciplinaire de Recherche (Berthoud, 1984, 27) dramatiquement nécessaire, les quelques analyses arrachées grâce à certaines relations personnelles privilégiées mais au combien précaires souvent liées à la mise au point d'une méthode archéométrique... La méthode mise au point et rendue au stade opérationnel, il arrive que l'archéologue n'obtienne plus la collaboration précédemment définie.

Comment accepter que des équipes de recherche (du CNRS, de l'Université, de la Sous-Direction de l'Archéologie ou de la Direction des Musées de France) censées faire de la recherche scientifique n'obtiennent pas, quasi automatiquement, la collaboration d'une autre équipe de ces mêmes instances quand les premières ont une problématique et un besoin impérieux qui, faute d'être honoré, entraînera la stagnation ou même l'arrêt des programmes en question? Problème de composition (et aussi de compétence dans ces domaines spécialisés) des commissions sans doute également...

Il manque aussi une politique cohérente de l'archéométrie (pas d'enseignement structuré, de structure d'évaluation des équipes et des programmes, pas de financement en rapport avec l'utili- 
sation normale des équipements, publications fort inégales).

Enfin le problème recherche/service des laboratoires reste toujours d'actualité. « ... Ces disciplines sont plus que jamais présentes dans l'archéologie contemporaine mais, une fois les techniques mises au point et les référentiels constitués, elles sortent du domaine expérimental pour être utilisées au sein de programmes archéologiques d'ensemble et les archéomètres participent à la constitution du discours archéologique proprement dit » (Tchernia, 1989, 2). L'archéométrie est ici reconnue. En fait, la recherche de base aboutie, il faut passer au service tout en poursuivant la recherche (mise au point de méthodes nouvelles ou adaptation) qui rebondit souvent au détour d'une question archéologique novatrice. Cette recherche poursuivie ne doit pas minimiser l'importance de la collaboration (terme peut-être préférable à celui de service...) à des programmes archéologiques.

« Pour y mettre bon ordre, en attendant la mise en place d'équipes d'archéométrie judicieusement réparties dans les diverses régions, on pourrait imaginer un régime de contrats passés entre les laboratoires existant aujourd'hui et les organismes qui financent les fouilles archéologiques » (Bouärd, 1987). L'effort est à faire aussi bien pour l'archéologie de sauvetage que pour la recherche programmée.

\section{POUR EN SORTIR}

"La percée réalisée par la France en ce domaine [l'archéologie du Moyen Age] constitue l'un des grands progrès de notre archéologie. Il est récent, il est fragile, il s'agit de le fortifier » (Goudineau, 1989, 25). Rééquilibrer le poids respectif de l'archéologie des différentes périodes apparaît bien nécessaire ; pour preuve, la place un peu dérisoire laissée au moyen âge et aux époques modernes dans l'exposition "Archéologie de la France, 30 ans de découvertes »... Reconsidérer l'archéologie de recherche passée à l'arrière-plan du " sauvetage " par compression budgétaire (ou autre politique) nécessite une dotation décente en dehors du financement de la recherche qui est entreprise normalement sur les sauvetages.

Pour faciliter les rapports archéologue/archéomètre et donner un peu d'esprit critique aux premiers, il faut intensifier leur formation aux méthodes des seconds et au traitement statistique, et vice versa.

Enfin donner une suite aux rapports (par ex. Berthoud, 1984) sur la situation de l'archéométrie.
«... Aucune suite officielle n'a été donnée... En l'espace d'un plan quinquennal, le doublement (tenu pour minimum) des moyens humains n'aura toujours pas été engagé, les laboratoires manquants n'existent toujours pas et aucune politique de formation n'a vraiment été définie... » (Ligot, 1989). A quand la reconnaissance de l'archéométrie traduite par un enseignement universitaire organisé ? Une structure d'évaluation des laboratoires et des programmes ayant une incidence sur les crédits rendrait sans doute de grands services. En découlerait aussi une littérature plus rigoureuse... A quand l'organisation d'un réseau de laboratoires pérennes et pluridisciplinaires, une politique de recherche jumelée à une collaboration des laboratoires aux programmes archéologiques pour une meilleure approche des sociétés anciennes?

La finalité de l'archéométrie (Démians d'Archimbaud, 1980, 21) : " un instrument efficace et commode au service de la recherche archéologique ».

Jacques THIRIOT

Chargé de recherche au CNRS ERA n ${ }^{\circ} 6$ d'Aix-en-Provence

BERTHOUd T. (1984) - Les Sciences de la nature et l'archéologie. Rapport sur la situation de la recherche française. 1984.

BouÄrd M. (de), MEYer N. et RANDOIN B. (1987) - Le Traitement de la céramique, La céramique ( $V^{e}-X I X^{e} s$. $)$, fabrication, commercialisation, utilisation, Paris, 1985. Caen, 1987, pp. 9-15.

Démians d'Archimbaud G. et Picon M. (1980) - Les Céramiques médiévales en France méditerranéenne. Recherches archéologiques et de laboratoire, La Céramique médiévale en Méditerranée occidentale, Valbonne, 1978. Paris, 1980, pp. $15-42$.

Démians d'Archimbaud G. et Picon M. (1987) - Céramiques d'habitat. Réflexion critique sur les données acquises lors des fouilles de Rougiers, La Céramique $\left(V^{e}-X I X^{e} s\right.$.), fabrication, commercialisation, utilisation, Paris, 1985. Caen, 1987, pp. 245258.

GoudineAu Ch. et GuILAINE J. (1989) - Les transformations de l'archéologie de la France, Archéologie de la France. 30 ans de découvertes. Catalogue d'exposition, Paris, 1989. Paris, 1989, pp. 18-28.

Ligot J. (1989) - Archéologie et archéométrie, Archéologie de la France. 30 ans de découvertes. Catalogue d'exposition, Paris, 1989. Paris, 1989 , p. 465.

PiCON M. (1989 a) - Caractérisation chimique des matériaux archéologiques. Stage de céramologie, Valbonne, 1989.

Picon M. (1989 b) - Quel avenir pour la céramologie de laboratoire? Archéologie Médiévale, XIX, 1989, pp. 243-254.

TCHERNiA A. et AudouZE F. (1989) - Développer une archéologie de recherche et préserver le patrimoine, Archéologie en France métropolitaine. Le Courrier du CNRS (dossiers scientifiques), 73, 1989, pp. 1-2.

WIDEMANN F. (1980) - Études analytiques de provenances pour les céramiques, Dossiers de l'archéologie, 42, 1980, pp. 28-38. 